\title{
The Influence of Infrasounds on the Electrocardiograph Patterns in Humans
}

\author{
C. KASPRZAK* \\ Department of Mechanics and Vibroacoustics, Faculty of Mechanical Engineering and Robotics \\ AGH University of Science and Technology, al. Mickiewicza 30, 30-059 Kraków, Poland
}

This study investigates how infrasound exposure should influence the electrocardiograph (ECG) patterns and the functioning of the cardiovascular system. Infrasound waves are widespread and common and now perceptible in the working environment (particularly in industry) as well as in the recreation grounds (natural sources of infrasounds). the main sources of infrasounds include heavy machines, transport and materials handling installations, as well as natural phenomena, such as blowing winds, storms or flowing waters. Research work has now established that low-frequency noise affects the morphology of the electric biopotentials of human brain and influence the blood pressure, the level of electrodermal activity and visual-motional coordination in humans. the effects of infrasounds on fluctuations of the ECG patterns have received little attention so far. This study summarises the research data to determine how low-frequency noise with frequency $f=7 \mathrm{~Hz}$ and the acoustic pressure level $S P L=120 \mathrm{~dB}$ (HP) should influence the ECG patterns. Experiments were performed on a group of 33 participants. Thus obtained experimental data indicate that infrasound exposure leads to major changes in the functioning of human heart.

PACS numbers: 87.50.Y-

\section{Introduction}

Infrasound waves are widespread and now perceptible in the working environment (particularly in industry) as well as in the recreation grounds (natural sources of infrasounds). The main sources of infrasounds include heavy machines, transport and materials handling installations, as well as natural phenomena, such as blowing winds, storms or flowing waters $[1,2]$.

Research on low-frequency noise began in France during the First World War, prompted by the search for their potential military applications. During the World War II the first well-documented experiments were performed to explore how infrasounds and low-frequency sounds affected humans. In 1954 the State Research Centre in Marseilles (France) began to systematically collect data on biological effects of infrasounds on humans.

Research data gathered to date reveal that the impacts of low-frequency sounds on humans are decidedly negative, though not fully explained yet [3]. The effects include the changes in the functioning of the nervous, respiratory, digestive and circulatory systems. The term "nuisance" is frequently used in literature when describing the effects that infrasounds have on humans. Research data and experimental results are sometimes ambiguous, even contradictory. Besides, they fail to account for low-energy waves which might positively impact on humans as long as the exposure time is short $[4,5]$.

\footnotetext{
* corresponding author; e-mail: ckasp@agh.edu.pl
}

The effects of low-frequency acoustic waves on human biopotentials are addressed in few publications only. Few research programs involved qualitative and quantitative analyses of biopotentials patterns of the human brain and of the activation level induced by acoustic stimuli.

The literature on the subject reports several types of the human body's response to low-frequency noise exposure. However, no straightforward correlation has been found between the subjective assessment and fluctuation of functional body parameters, understood to be the selected physiological and psychological parameters and selected estimates of biological signals. Results of pilot tests conducted by the Author suggest that there exist clearly specified wave frequencies and amplitudes such that their biomedical applications appear to be practicable and fully justified. The application of low-frequency sound waves with precisely controlled parameters (frequency, acoustic pressure level, exposure time) might lead to lowered activation level of the autonomic nervous system) or change the morphology of the electrocardiograph pattern. In certain cases these effects prove beneficial, which might be utilised in medicine, in diagnostics and in therapy.

Apart from specific aural, resonance or vestibular effects during the low-frequency noise exposure, of particular importance are the effects that low frequency noise noise has on the autonomic nervous system. Its dysfunction leads to malfunctioning and disorders of the respiratory, digestive, endocrine and nervous systems. These adverse impacts are revealed by disorders of the central nervous systems, characteristic of the lowered waking 
state. These changes are correlated with disorders of the respiratory and circulatory systems (reduced pulse rate and breathing rate). Frequency proves to be of less importance here than when aural or resonance effects are considered [6]. Physiological effects are treated as indirect consequences since they were first "processed" by the nervous system. The nervous system responds to infrasounds as a "system", in other words, emotional effects coincide with vegetative effects. These effects are functional and hence passing: trembling fingers, disturbed heart action.

Physiological, metabolic, psychomotor effects are stimulated by the actions of the nervous system. They become more intense with an increased acoustic pressure level. At the pressure level $100 \mathrm{~dB}$ the pulse rate and the breathing rate decrease and the amount of adrenaline expelled from the system with urine is reduced. These might be the confirmation of the subjective feeling of tiredness and sleepiness. Physiological tests conducted by Landström [7] revealed that at the pressure $110 \mathrm{~dB}$ the waking state is reduced, replaced by tiredness and drowsiness (evidenced by the ECG patterns) and the activity of the circulatory system gets reduced. According to Landström, these are the consequences of disrupted action of the nervous system, particularly of the reticular formation, caused by stimulation of the auditory tract. The impacts of infrasound noise on the nervous system are also revealed by experiments [8] testing the effects of infrasound exposure in sleep. Similar results were obtained when testing the effects of infrasounds [9] on psychological and physiological parameters and bioelectric patterns of the human brain $[10,11]$.

When the acoustic pressure levels are in excess of $130 \mathrm{~dB}$, the accompanying vegetative disorders are fairly unambiguous. Landström showed that hydrochloric acid production in the stomach is reduced, the breathing rate becomes slower and the feeling of drowsiness prevails accompanied by changes in the function of the circulatory system: lowered systolic pressure and increased diastolic pressure, reduced secretion of cortisone and adrenaline. On the other hand, infrasounds with the acoustic pressure level $135 \mathrm{~dB}$ and frequency 5 and $10 \mathrm{~Hz}$ [12] led to emotional tension, tiredness, drowsiness, the feeling of pressure in ears. They also produced arrhythmia and disturbances of heart action, revealed as the lower force of the heart muscle, variations of the cardiac contraction rate, disturbances of brain hemodynamics - increase of the reographic wave amplitude and prolonging of the anacrotic phase. Experiments on laboratory animals [13] revealed the changes of bioelectric activity of cortical and subcortical brain structures. Of major interest were the registered changes in the composition of nucleic acids in receptor cells in the cochlea and in the cell volume.

\section{Experimental procedure}

The group of participants in the experimental program included 33 healthy men and women, aged 20-30 years.
The experimental procedure consisted in a 20 min exposure to the acoustic wave with the precisely controlled parameters: $f=7 \mathrm{~Hz}$ and $S P L=120 \mathrm{~dB}(\mathrm{HP})$.

Tested parameters (dependent variables) included: bioelectric potentials of heart, diastolic and systolic pressure and the pulse rate. Participants who qualified for the tests did not report any earlier low frequency noise exposure, and had not previously suffered from diseases of hearing organs or any other chronic illnesses.

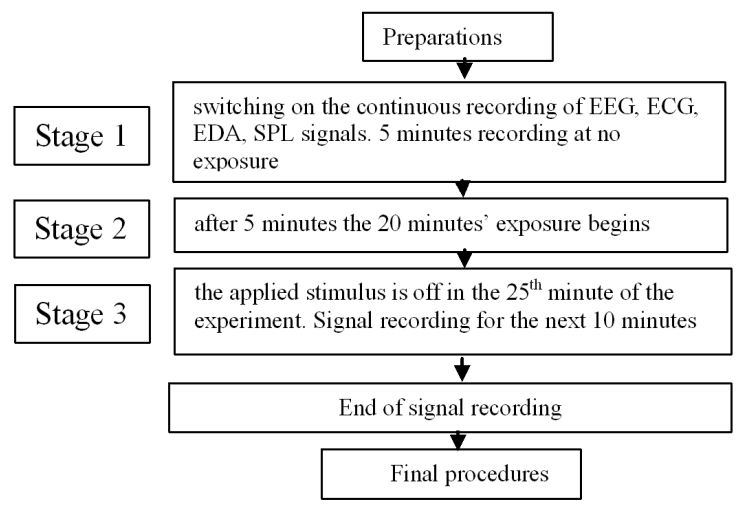

The experimental setup incorporates:

- circuit for generating acoustic signals,

- circuit for measuring the acoustic conditions,

- circuit for bioelectric signal recording.

This configuration enables the application of infrasound exposure in laboratory conditions (with precisely controlled parameters) and measurement and analysis of the effects that low frequency noise has on functional parameters of the human body.

The ECG recording circuit comprises a standard pre-amplifier SAM25 (Micromed) and dedicated software for recording of bioelectric potentials. ECG electrodes are attached at the heart's level, using an elastic belt. The reference electrode is attached on the right-hand side of the test participant, about $10 \mathrm{~cm}$ below the measurement electrode. These are all surface electrodes, $1.3 \mathrm{~cm}$ in diameter. To make sure the electrodes fitted tightly to the skin, a ECG gel Via Dan G was applied on the skin. A well-configured measurement circuit enabled the constant acquisition of biopotential signals (35 min during the main test) and digital recording.

Measurements of systolic pressure and of the pulse rate were repeated twice: before and after the experiment.

\section{Results}

The effects of infrasound exposure on the action of the circulatory system were investigated by two methods: the quantitative method to determine the fluctuation of functional parameters: systolic and diastolic pressure and the pulse rate, and the qualitative method to characterise the 
changes in the ECG pattern during the experiment. Accordingly, the ECG signal was subjected to the frequency analysis and the results are summarised on the graph.

Qualitative analyses of the measured diastolic and systolic pressure and of the pulse rate were performed twice: before and after the experiment. Results are summarised in Table.

- S_BEFORE - systolic pressure before the test,
- S_AFTER - systolic pressure after the test,

- R_BEFORE - diastolic pressure before the test,

- R_AFTER - diastolic pressure after the test,

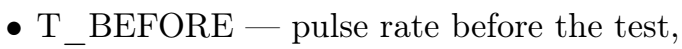

- T_AFTER - pulse rate after the test.

TABLE

Analyses of the measured diastolic and systolic pressure and of the pulse rate.

\begin{tabular}{l|c|c|c|c|c|c|c|c}
\hline \hline & $\begin{array}{c}\text { Mean } \\
\text { value }\end{array}$ & $\begin{array}{c}\text { Standard } \\
\text { deviation }\end{array}$ & $N$ & Difference & $\begin{array}{c}\text { Standard } \\
\text { deviation }\end{array}$ & $t$ & $d f$ & $p$ \\
\hline S_BEFORE & 122.65 & 14.59 & & & & & & \\
S_AFTER & 120.93 & 12.34 & 32 & 1.71 & 10.38 & 0.93 & 31 & 0.35 \\
R_BEFORE & 73.03 & 8.64 & & & & & & \\
R_AFTER & 75.65 & 6.10 & 32 & -2.62 & 8.22 & -1.80 & 31 & 0.08 \\
T_BEFORE & $\mathbf{7 2 . 9 3}$ & $\mathbf{1 0 . 6 1}$ & & & & & & \\
T_AFTER & $\mathbf{6 6 . 7 1}$ & $\mathbf{9 . 6 1}$ & $\mathbf{3 2}$ & $\mathbf{6 . 2 1 8 8}$ & $\mathbf{7 . 2 7}$ & $\mathbf{4 . 8 3}$ & $\mathbf{3 1}$ & $\mathbf{0 . 0 0 0 0 3 4}$ \\
\hline
\end{tabular}

where: $N$ - group size, $t$ - test value, $d f-$ degrees of freedom, $p$ - statistical significance.

The analyses reveal the statistically significant reduction of the pulse rate from the initial level $73[1 / \mathrm{min}]$ to $67[1 / \mathrm{min}]$. At the same time diastolic pressure went up and systolic pressure went down, both changes being statistically insignificant.

The qualitative analysis used the Fourrier transform to handle $10 \mathrm{~s}$ ECG signals and to provide the graphic display (Fig. 2). On the axes are the test time (35 min), frequency (from 0.5 to $2 \mathrm{~Hz}$ ) and on the vertical axis is the amplitude of the signal $\left(\mathrm{mV}^{2} / \mathrm{Hz}\right)$.

The analysis of qualitative changes of the ECG pattern revealed the occurrence of a phenomenon known as "stabilisation of the heart action" during the infrasound exposure. This phenomenon was observed in $30 \%$ of test participants and the amplitude become apparent, too. It was found out that the rate of heart action tends to decrease throughout the experiment, which is corroborated by pulse rate measurements taken before and after the experiment.

\section{Discussion}

The analyses of the effects of infrasound exposure on the functioning of the circulatory system revealed a statistically significant reduction of the pulse rate whilst the observed increase of the diastolic pressure and increase of the systolic pressure appear to be statistically insignificant.

Qualitative analyses of infrasound exposure and its effects on the ECG pattern reveal that the rate of heart

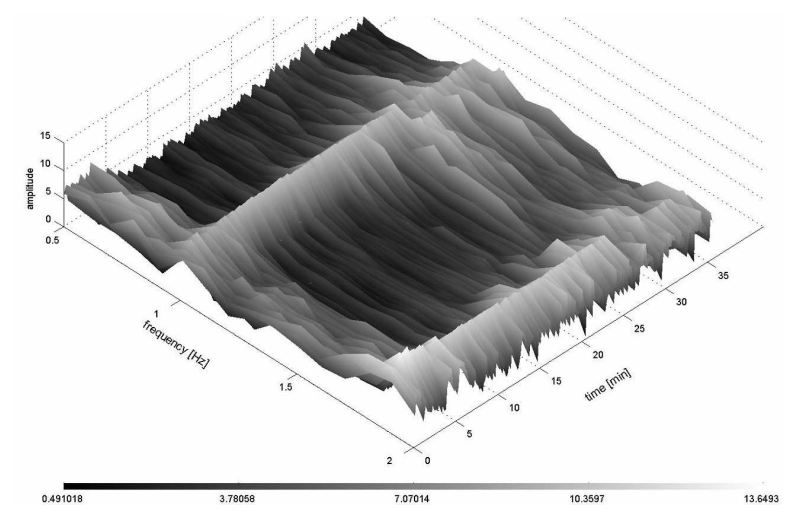

Fig. 2. Changes of the ECG pattern.

action tends to stabilise during the infrasound exposure, this phenomenon was registered in $20 \%$ of test participants. The analyses, therefore, seem to evidence the effects that infrasound exposure has on the ECG signal patterns.

\section{References}

[1] Z. Damijan, C. Kasprzak, Pol. J. of Environmental Studies 13, (2004).

[2] M.A. Garces, Geoscience and Remote Sensing Symposium, , (2000).

[3] H. Møller, J. Andresen, Proc. Internoise, 911 (1984). 
[4] N. Broner, J. Sound Vibr. 58, 483 (1978).

[5] T. Wigram, Thesis, St. Georges hospital, medical school London university, 2000.

[6] S. Yamada, M. Sueki, S. Hagiwara, T. Watanabe, T. Kosaka, J. Low Frequency Noise and Vibration 19, 130 (1991).

[7] U. Landsröm, P. Pelmer, J. Low Frequency Noise and Vibration 12, 72 (1993).

[8] K. Persson, Noise and Health, 6, 87 (2004).

[9] Y. Chen, Q. Huang, S. Hanmin, J. Low Frequency Noise, Vibration Active Control 71, (2004).
[10] C. Kasprzak, Z. Damijan, R. Panuszka, Polish J. Environmental Studies 13, (2004).

[11] C. Kasprzak, Pol. J. Environmental Studies, Vol. 16 3B, 217 (2007).

[12] H. Karpova, S. Aleksiejev, V. Erochin, Gigiena Truda i Profiesjonalnyjen Zabolevanija 10, (1979).

[13] C. Rice, D. Robinson, Proc. 15th Int. Congress Acoustics, Ed. M. Newman, Acoustical Society of Norway, Trondheim 1995, p. 263. 